\title{
Qualidade dos serviços de saúde: uma avaliação na Atenção Primária em um município de Goiás
}

\author{
Quality of health services: an assessment of Primary Care in a city of Goiás
}
Calidad de los servicios de salud: una evaluación de la Atención Primaria en una ciudad de Goiás

Ricardo Rabelo Aguilar ${ }^{1 *}$, Isabella Françoise Teles ${ }^{1}$, Karla Cristina Naves de Carvalho.

\begin{abstract}
RESUMO
Objetivo: Avaliar a qualidade da Atenção Primária à Saúde (APS) em um município de Goiás através do cálculo dos escores dos atributos essenciais e derivados dos serviços de saúde, identificando os atributos de menor e maior escore, bem como, o escore geral da APS. Métodos: Trata-se de um estudo de campo descritivo, quantitativo e transversal desenvolvido durante o mês de março de 2021, abordando 32 profissionais atuantes nas unidades básicas de saúde de um município de Goiás, sendo 22 médicos e 10 enfermeiros, que responderam devidamente o questionário PCATool-Brasil 2020. Resultados: O atributo acesso de primeiro contato (acessibilidade) alcançou o menor valor de escore $(3,41)$, enquanto a integralidade (serviços disponíveis), o maior $(7,59)$. A qualidade da APS no município foi avaliada como pouco orientada às necessidades de sua população, com escore geral de 6,19. Conclusão: Apesar de realizado em contexto pandêmico, o estudo identificou falhas e acertos no cuidado fornecido aos pacientes, com o potencial de proporcionar o planejamento e a adoção de condutas preventivas e assistenciais mais eficazes.
\end{abstract}

Palavras-chave: Acesso aos serviços de saúde, Atenção primária à saúde, Avaliação dos serviços de saúde, Integralidade em saúde, Qualidade da assistência à saúde.

\begin{abstract}
Objective: To assess the quality of Primary Health Care (PHC) in a city of Goiás by calculating the scores of essential and derived attributes from health services, identifying the attributes with the lowest and highest scores, as well as the overall score of PHC. Methods: This is a descriptive, quantitative and cross-sectional field study developed during the month of March 2021, approaching 32 professionals working in basic health units in a city of Goiás, being 22 doctors and 10 nurses, who duly answered the PCATool- Brazil 2020 questionnaire. Results: The attribute first contact accessibility reached the lowest score value (3.41), while integrality (available services) had the highest (7.59). The quality of PHC in the city was assessed as poorly oriented to the needs of its population, with an overall score of 6.19. Conclusion: Despite being carried out in a pandemic context, the study identified flaws and successes in the care provided to patients, with the potential to provide planning and adoption of more effective preventive and care measures.
\end{abstract}

Keywords: Health services accessibility, Primary health care, Health services research, Integrality in health, Quality of health care.

\footnotetext{
1 Universidade Evangélica de Goiás (UniEvangélica), Anápolis - GO.

*E-mail: ricardorabeloaguilar@gmail.com
}

O estudo faz parte do Programa de Iniciação Científica (PIBIC) da Universidade Evangélica de Goiás (UniEvangélica), 2020-2021. 


\section{RESUMEN}

Objetivo: Evaluar la calidad de la Atención Primaria de Salud (APS) en una ciudad de Goiás mediante el cálculo de las puntuaciones de los atributos esenciales y los derivados de los servicios de salud, identificando los atributos con menor y mayor puntaje, así como el puntaje general de APS. Métodos: Se trata de un estudio de campo descriptivo, cuantitativo y transversal desarrollado durante el mes de marzo de 2021, acercándose a 32 profesionales que laboran en las unidades básicas de salud de una ciudad de Goiás, siendo 22 médicos y 10 enfermeras, quienes respondieron debidamente el cuestionario PCAToolBrasil 2020. Resultados: El atributo accesibilidad y primer contacto alcanzó el valor de puntuación más bajo $(3,41)$, mientras que la integralidad (servicios disponibles) tuvo el valor más alto $(7,59)$. La calidad de la APS en la ciudad se evaluó como mal orientada a las necesidades de su población, con un puntaje general de 6,19. Conclusión: Aunque logrado en un contexto pandémico, el estudio identificó fallas y éxitos en la atención proporcionada a los pacientes, con el potencial de brindar la planificación y adopción de medidas preventivas y asistenciales más efectivas.

Palabras clave: Accesibilidad a los servicios de salud, Atención primaria de salud, Investigación sobre servicios de salud, Integralidad en salud, Calidad de la atención de salud.

\section{INTRODUÇÃO}

A Atenção Primária à Saúde (APS) configura-se como um modelo de assistência que visa ampliar e efetivar o acesso da população ao sistema de saúde bem como aos serviços por ele ofertados. Caracterizase por um conjunto de ações individuais e coletivas que buscam desenvolver uma atenção integral, universal e equânime capaz de impactar na situação de saúde, na autonomia dos indivíduos, nos determinantes e condicionantes de saúde das populações sob seus cuidados (STARFIELD B, 2002; FAUSTO MCR e MATTA GC, 2007; REIS RS, et al., 2013; MINISTÉRIO DA SAÚDE, 2017).

Considerada a principal porta de entrada ao sistema público de saúde brasileiro, a APS baseia-se na assistência às principais demandas de saúde das populações, de modo a otimizar a resolutividade dos principais problemas das coletividades. Dessa forma, a dinâmica do acolhimento consiste na promoção, prevenção, diagnóstico, tratamento adequado, reabilitação e acompanhamento longitudinal dos usuários durante todas as fases de suas vidas (MENDES EV, 2011).

De acordo com Starfield B (2002), a APS é formada por seis atributos, divididos em essenciais - acesso de primeiro contato (acessibilidade), longitudinalidade, coordenação (integração de cuidados e sistemas de informações) e integralidade (serviços disponíveis e prestados) e derivados - orientação familiar e orientação comunitária. Dentro dessa perspectiva, cada atributo é composto por fatores que os incorporam às diretrizes do Sistema Único de Saúde (SUS) de forma a promover um modelo de cuidado democrático e inclusivo.

O acesso de primeiro contato incorpora formas de acessibilidade e utilização dos serviços de saúde pela população. Uma vez acolhido pela APS, o indivíduo entra em contato com um modelo de cuidado regular e continuado pela equipe de saúde, a longitudinalidade. A coordenação da atenção, por sua vez, relaciona-se à capacidade de garantir a continuidade da atenção no interior da rede de serviços disponibilizados, bem como promover a articulação entre os diversos serviços e ações de saúde. E nesse sentido, surge a integralidade que consiste no reconhecimento de padrões sociais e demandas locais, ofertando serviços que atendam às necessidades mais comuns desta população (OLIVEIRA MAC e PEREIRA IC, 2013; SILVA KF, 2017; PUCCI VR, 2017).

Por outro lado, as orientações familiar e comunitária implicam, respectivamente, a avaliação de eixos familiares como sujeitos da atenção e ao reconhecimento das necessidades coletivas, considerando o contexto territorial, cultural e socioeconômico. Por conseguinte, o propósito da APS é proporcionar o acesso à assistência integral, por meio de condutas coordenadas de acordo com a demanda familiar e comunitária ao longo do tempo (ANDERSON MIP, et al., 2016; PUCCI VR, 2017). 
Desse modo, haja vista a grande influência dos atributos da APS no processo de organização do sistema de saúde, torna-se fundamental a produção de conhecimentos que possibilitem a avaliação da qualidade dos cuidados oferecidos à população. No contexto das comunidades, a avaliação da APS apresenta-se como forma de identificar dificuldades no desenvolvimento de ações específicas, analisar os resultados das políticas de saúde implementadas e auxiliar no processo decisório da gestão em saúde e prestação de serviços, permitindo a implementação de medidas que levem ao desenvolvimento de serviços melhor orientados para a APS, congruentes às realidades locais (MINISTÉRIO DA SAÚDE, 2005; SCHMIDT MI, et al., 2011; PIRES DA, 2012).

Sendo assim, a fim de realizar uma avaliação da qualidade da APS, o instrumento Primary Care Assessment Tool (PCATool-Brasil 2020) é considerado por Stein AT (2013) como a melhor ferramenta para a identificação dos atributos que exigem reafirmação ou reformulação na busca por excelência no cuidado (STARFIELD B, 2002; MINISTÉRIO DA SAÚDE, 2020). Por meio dele obtêm-se escores que possibilitam estabelecer o grau de orientação à APS, viabilizando a comparação de atributos, a identificação de componentes que necessitam de intervenções, bem como, daqueles que já se encontram em níveis elevados de qualidade e desenvolvimento. É fundamental para que gestores e pesquisadores possam estabelecer metas e correlacionar a presença dos atributos e a efetividade da atenção tanto em nível individual quanto coletivo (HARZHEIM E, et al., 2013; STEIN AT, 2013; MINISTÉRIO DA SAÚDE, 2020).

Neste contexto, o presente estudo objetivou avaliar a qualidade da atenção primária em um município de Goiás, a partir de entrevistas aos profissionais médicos e enfermeiros, por meio do instrumento PCAToolBrasil 2020. Serão discutidos somente os atributos que alcançarem o menor e o maior escore, assim como o escore geral da APS.

\section{MÉTODOS}

Trata-se de um estudo transversal, descritivo, de abordagem quantitativa das características organizacionais e do desempenho dos serviços das Equipes de Saúde da Família. Possui caráter avaliativo dos atributos da APS, tendo sido realizado em março de 2021 em um município de Goiás. Para isso, foi aplicado o questionário de avaliação da APS (PCATool-Brasil 2020 - versão extensa para profissionais), disponibilizado pelo Ministério da Saúde (MINISTÉRIO DA SAÚDE, 2020).

Sabe-se que no início do ano de 2020, o Sars-CoV-2 provocou sérias alterações nos sistemas de saúde mundiais e durante o mês de março daquele ano foi decretado estado de emergência de saúde pública em todo o território brasileiro. A pandemia causada pelo novo coronavírus (Covid-19) trouxe consigo profundas fragmentações na qualidade e distribuição dos serviços ofertados pelo SUS nacionalmente, o que se refletiu de forma equivalente nesse município.

Em meio à gravidade da situação e de sua progressão exponencial em número de casos e óbitos, o sistema de saúde precisou se articular de forma a corroborar na assistência às vítimas dessa doença. $\mathrm{Na}$ Atenção Primária, isso se refletiu em Unidades Básicas de Saúde (UBS) que se reestruturaram em centros de referência para atendimento e tratamento de casos suspeitos ou confirmados de Covid-19. Nesse sentido, as populações adscritas que estavam sob a responsabilidade desses serviços ficaram fragilizadas quanto à continuidade do cuidado e foram referenciadas a outras unidades.

As unidades não convertidas em centros de referência ao Covid-19, também enfrentaram desafios como número insuficiente de insumos, precariedade de infraestrutura e sobrecarga de demandas populacionais. Assim sendo, apesar da necessidade de organizar novas equipes, tal articulação não conseguiu se desenvolver de forma integral, formando equipes de saúde incompletas, sem profissionais enfermeiros, técnicos, auxiliares ou até mesmo médicos.

Diante dessa inesperada perspectiva, a população do estudo foi composta por profissionais atuantes nas equipes de saúde da família que recepcionam o programa de residência médica de Medicina de Família e Comunidade nesse município. A seleção dessa amostra justificou-se pela intenção de garantir que os participantes estivessem inseridos em equipes completas e que permaneceram atendendo demandas não relacionadas à pandemia do novo coronavírus. 
Foram escolhidos como sujeitos da pesquisa profissionais médicos e enfermeiros, na qual foram adotados como critérios de inclusão aqueles que participavam regularmente das atividades da UBS, se consideravam cientes dos serviços e da realidade local e que se dispuseram a preencher o questionário. Ao passo que profissionais em recesso, férias ou licença médica no período e os que se recusaram a participar da pesquisa ou não assinaram o Termo de Consentimento Livre e Esclarecido (TCLE) foram excluídos do estudo.

O preenchimento dos questionários foi realizado no ambiente de trabalho dos profissionais, mediante aprovação do Comitê de Ética em Pesquisa da Universidade Evangélica de Goiás (UniEvangélica) $n$. 4.165.477 (CAAE 34356620.5.0000.5076), da Secretaria Municipal de Saúde e dos responsáveis administrativos de cada UBS. Os momentos para emprego dos questionários ocorreram com ajustes à carga horária dos participantes, de forma a ocupar minimamente o horário de trabalho e, assim, não prejudicar a assistência à saúde prestada. O tempo estimado de resolução dos questionários foi de 20 minutos, podendo ser flexibilizado à disponibilidade individual.

Dentre os riscos prováveis da pesquisa detectou-se o possível constrangimento dos participantes e, com o intuito de minimizá-lo, os questionários foram respondidos em ambiente adequado sem a presença de outras pessoas e não foram identificados com dados pessoais dos participantes. Tal estratégia buscou assegurar a confidencialidade e a veracidade das informações colhidas. Além disso, como benefício direto da pesquisa observou-se a oportunidade de estabelecer um canal de comunicação entre os participantes e os entrevistadores, visando identificar aspectos estruturais e processuais que exigem reafirmação ou reformulação.

Durante o período da coleta, realizada em março de 2021, foram considerados 41 profissionais elegíveis, dos quais, três recusaram-se a participar e outros seis não foram encontrados após duas tentativas de aplicação dos questionários em dias diferentes de funcionamento regular de sua respectiva UBS. Assim sendo, após a aplicação dos critérios de inclusão e exclusão, a amostra final obtida foi de 32 profissionais, distribuídos em seis unidades de saúde.

O PCATool-Brasil 2020 é formado por 111 itens subdivididos em 8 componentes relacionados aos atributos da APS e a análise estatística dos questionários foi desenvolvida conforme as prerrogativas do instrumento. Os itens do questionário possuem cinco possíveis opções de resposta: "com certeza sim", "provavelmente sim", "provavelmente não", "com certeza não" e "não sei/ não lembro" (MINISTÉRIO DA SAÚDE, 2020).

O cálculo de escore dos atributos da APS para cada pessoa entrevistada depende da quantidade de itens indicados com valores considerados ausentes, correspondentes a itens sem resposta ou com respostas "não sei/não lembro". Apenas foram considerados os escores dos participantes em que o percentual dos itens de valores ausentes foi inferior a $50 \%$ da quantidade total de itens do atributo. Nesses casos, a pontuação de tais itens foi convertida para resposta "provavelmente não", de modo a minimizar a perda de dados e classificar negativamente as características do serviço de saúde que podem não ser de conhecimento da pessoa entrevistada. Porém, na ocorrência de percentual superior ou igual a $50 \%$, não foram calculados os escores referentes ao atributo correspondente, permanecendo em branco no banco de dados e considerados pelos pesquisadores como "indevidamente preenchidos" (MINISTÉRIO DA SAÚDE, 2020).

Os escores para cada um dos atributos foram calculados pela média aritmética simples das respostas dos itens que os compõe e através dos escores, obteve-se a pontuação equivalente ao escore geral da APS no município. Além disso, novas médias aritméticas simples foram calculadas verificando a perspectiva de médicos e enfermeiros, de forma a avaliar a qualidade da atenção do ponto de vista de cada grupo de profissionais. E a fim de facilitar a análise dos resultados, cada escore foi convertido em valores que variam de 0 a 10, de acordo com a fórmula prevista no PCATool-Brasil 2020 (MINISTÉRIO DA SAÚDE, 2020).

Diante do exposto, as pontuações obtidas foram classificadas em alta (escore $\geq 6,6$ ) e baixa (escore < 6,6), com a primeira revelando serviços melhor orientados para a APS e a última demonstrando a deficiência na execução de determinado atributo, destacando a necessidade de intervenções resolutivas (MINISTÉRIO DA SAÚDE, 2020). 
Os resultados foram tabulados em banco de dados do programa de estatísticas do Excel para possibilitar a realização de cálculos de médias e o desenvolvimento de tabelas para análises estatísticas, sendo utilizado o intervalo de confiança de $95 \%$.

\section{RESULTADOS}

A Tabela 1 evidencia os escores gerais de cada um dos atributos essenciais e derivados dos serviços de APS, considerando somente os escores dos participantes em que o percentual dos itens de valores ausentes foi inferior a $50 \%$ da quantidade total de itens do atributo. Nesse contexto, o atributo essencial Acesso de Primeiro Contato (Acessibilidade) foi devidamente avaliado por 32 profissionais, obtendo a menor média entre os atributos, com valor de 3,41 e desvio padrão de 1,25. Concomitantemente, o atributo essencial Integralidade (Serviços Disponíveis) foi devidamente avaliado por 32 profissionais, obtendo a maior média entre os atributos, alcançando o valor de 7,59 e desvio padrão de 1,16.

De acordo com as considerações do instrumento utilizado, pode-se afirmar que os atributos Coordenação (Sistema de Informações), Integralidade (Serviços Disponíveis), Integralidade (Serviços Prestados) e Orientação Familiar obtiveram altas pontuações nos escores, inferindo a presença de componentes melhor orquestrados às necessidades das populações assistidas. Todavia, os atributos Acesso de Primeiro Contato (Acessibilidade), Longitudinalidade, Coordenação (Integração de Cuidados) e Orientação Comunitária obtiveram baixas pontuações, revelando possíveis deficiências na execução destes atributos (Tabela 1).

Tabela 1 - Escore dos Atributos Essenciais e Derivados dos Serviços de APS.

\begin{tabular}{cccc}
\hline Atributos & N & Média & Desvio Padrão \\
\hline Acessibilidade & 32 & 3,41 & 1,25 \\
Longitudinalidade & 32 & 5,93 & 1,24 \\
Coordenação (Integração de Cuidados) & 32 & 6,43 & 1,32 \\
Coordenação (Sistema de Informações) & 31 & 6,62 & 1,56 \\
Integralidade (Serviços Disponíveis) & 32 & 7,59 & 1,16 \\
Integralidade (Serviços Prestados) & 32 & 7,03 & 1,76 \\
Orientação Familiar & 32 & 7,00 & 1,61 \\
Orientação Comunitária & 29 & 5,49 & 1,56 \\
\hline
\end{tabular}

Fonte: Aguilar RR, et al., 2021.

A Tabela 2 apresenta o escore geral da APS e as perspectivas de médicos e enfermeiros avaliadas separadamente. Nessa ótica, o escore geral da APS obteve média igual a 6,19 $(<6,6)$, demonstrando qualidade dos serviços de saúde incongruente às demandas populacionais. É perceptível ainda, que há consenso entre os profissionais entrevistados, haja vista a baixa pontuação atribuída tanto por médicos como por enfermeiros.

Tabela 2 - Escore Geral da APS sob a Perspectiva dos Profissionais Médicos e Enfermeiros.

\begin{tabular}{cccc}
\hline Escore & N & Média & Desvio Padrão \\
\hline Médicos & 22 & 6,23 & 0,85 \\
Enfermeiros & 10 & 6,12 & 0,98 \\
Escore Geral & 32 & 6,19 & 0,88 \\
\hline
\end{tabular}

Fonte: Aguilar RR, et al., 2021.

\section{DISCUSSÃO}

A avaliação dos atributos da APS é fundamental para a identificação das características, deficiências e fortalezas de cada serviço, de forma a promover um planejamento qualificado de ações que aumentem a efetividade da atenção à saúde (TANAKA OY e TAMAKI EM, 2012). Nesse contexto, a análise dos resultados alcançados evidencia que o atributo Acesso de Primeiro Contato (Acessibilidade) foi o de menor valor médio $(3,41)$ e desvio padrão estatisticamente baixo $(1,25)$, o que demonstra que os participantes da pesquisa têm opiniões semelhantes a respeito do atributo em questão (MINISTÉRIO DA SAÚDE, 2020). 
No que diz respeito ao atributo supracitado, Donabedian A (1973) defendia a ideia de que a acessibilidade estaria relacionada às características dos serviços e recursos de saúde que poderiam facilitar ou limitar sua utilização pelos usuários. Tal conceito se expande para além da entrada nos serviços, indicando também o nível de ajuste entre as demandas de cada população e a disponibilidade de recursos correspondentes.

Starfield B (2002) reforça que a acessibilidade se expressa como componente estrutural imprescindível para que a APS se estabeleça como porta de entrada dos sistemas de saúde. Afinal, uma vez consolidada, consegue solucionar a maioria das necessidades em saúde da população, reduzir a demanda por níveis de maior complexidade, racionalizar a utilização de recursos e potencializar a satisfação dos usuários (MINISTÉRIO DA SAÚDE, 2009).

Tendo em vista a avaliação da acessibilidade e a constatação da baixa orientação à APS, pode-se inferir a presença de diversas fragilidades relacionadas à assistência prestada. De acordo com os questionamentos presentes no instrumento PCATool-Brasil 2020, é possível deduzir que tais vulnerabilidades podem estar relacionadas à ausência de funcionamento das unidades de saúde em períodos noturnos ou em fins de semana, à dificuldade de disponibilizar atendimento rápido para pacientes com quadros agudos, bem como à escassez de ferramentas de comunicação em horários não comerciais (MINISTÉRIO DA SAÚDE, 2020).

Nesse sentido, faz-se necessário o estabelecimento de equipes que possam realizar revezamento de turnos de trabalho para a cobertura de horários e dias ainda não contemplados pelas unidades de saúde, de modo a ampliar as formas de acesso dos usuários. O turno estendido é fundamental para o atendimento de indivíduos que cumprem jornadas de trabalho em horários que coincidem com o período de funcionamento das unidades de saúde e apresentam dificuldades para comparecer às consultas, o que acarreta a busca por atendimento em outros níveis de atenção (NIED MM, et al., 2020).

Além disso, é essencial que haja otimização do processo de agendamento das consultas, com abertura de horários para atendimento de demandas espontâneas de baixa complexidade, que podem ser abordadas na APS e não fazem parte do escopo de atuação de Unidades de Pronto Atendimento (UPA). Ainda nesse contexto, o desenvolvimento de ferramentas de comunicação de fácil utilização pelos pacientes é importante para informar, sanar dúvidas, realizar agendamentos e verificar locais de atendimento próximos ao domicílio, de acordo com o nível de complexidade necessária para assistência da demanda em questão.

Paralelamente, a análise dos resultados alcançados demonstra que o atributo Integralidade (Serviços Disponíveis) foi o de maior valor médio $(7,59)$ e o menor desvio padrão $(1,16)$, evidenciando opiniões semelhantes entre os participantes do estudo.

Quanto a este atributo, Starfield B (2002) caracteriza-o como o conjunto de serviços ofertados pela APS suficientes para suprir as necessidades mais prevalentes da população. Dentro dessa perspectiva, a integralidade articula tanto âmbitos assistenciais quanto preventivos, de forma a garantir os direitos às ações assistenciais dos pacientes portadores de comorbidades e resguardar os indivíduos não portadores para que se beneficiem de ações preventivas (MATTOS RA, 2007; PUCCI VR, 2017).

No contexto da APS, a integralidade busca reconhecer a diversidade de necessidades em saúde de cada paciente, sua respectiva família e comunidade, disponibilizando recursos e serviços para assisti-los. Nesse cenário, a APS é responsável por garantir que o indivíduo receba todos os tipos de serviços dos quais necessita, ainda que alguns não sejam oferecidos por ela e precisem ser referenciados a serviços de níveis de atenção secundários ou terciários (PUCCI VR, 2017).

O destaque deste atributo está relacionado ao âmbito de serviços disponíveis pela APS, como aconselhamento relativo a questões nutricionais, planejamento familiar, abordagem de vícios, imunizações, sorologias, programas de assistência social, atendimento a saúde da criança, do adulto e do idoso, assistência odontológica, atendimento em saúde mental, pré-natal e pequenos procedimentos. Uma gama tão vasta de serviços oferecidos pela APS permite seu estabelecimento como referência no sistema de saúde brasileiro (MINISTÉRIO DA SAÚDE, 2020). 
Foi observado que o atributo Integralidade (Serviços Prestados) também obteve pontuação alta, com média de 7,03, mas sem ter alcançado escore equivalente ao atributo Integralidade (Serviços Disponíveis). Tal circunstância permite o questionamento quanto ao nível de acesso das populações aos serviços ofertados pelas unidades de saúde, reforçado pelo fato de o atributo acessibilidade ter alcançado o menor valor médio no estudo.

Esta problemática pode estar sendo orquestrada pela atual circunstância pandêmica de Covid-19 e a contínua articulação do sistema de saúde para a assistência às vítimas da doença. A adaptação do SUS ao tratamento da enfermidade sobrecarregou 0 atendimento de outras demandas comunitárias, comprometendo a acessibilidade e a continuidade do cuidado.

É possível inferir que o controle dos casos de Covid-19, a dissolução da quantidade de centros de referência e a redistribuição das populações entre as unidades de saúde desse município ocasionaria o aperfeiçoamento da acessibilidade aos serviços e a concomitante potencialização da integralidade, convertendo todos os serviços disponíveis em definitivamente ofertados.

Pode-se evidenciar ainda que a qualidade da assistência à saúde desse município foi avaliada como pouco orientada à APS e às necessidades de sua população. Esta afirmativa se mostra verdadeira, segundo a avaliação tanto de profissionais médicos quanto de enfermeiros, que avaliaram a assistência à saúde com as médias 6,23 e 6,12, respectivamente. Ao passo que o cálculo do escore geral da APS demonstrou valor médio de 6,19, também abaixo do ponto de corte estabelecido pelo PCATool-Brasil 2020, demonstrando a importância do planejamento e execução de intervenções governamentais que busquem o aprimoramento do modelo de atenção (MINISTÉRIO DA SAÚDE, 2020).

Haja vista a essência quantitativa do instrumento utilizado no estudo é possível inferir a existência de perdas informacionais relacionadas a aspectos subjetivos da assistência à saúde, que seriam mais bem avaliadas por metodologias de natureza qualitativa. Concomitantemente, o presente estudo considerou somente a realidade dos profissionais de saúde atuantes nas unidades básicas, sem avaliar a perspectiva dos indivíduos que usufruem dos serviços ofertados (HARZHEIM E, et al., 2006; HAUSER L, et al., 2013; PENSO JM, et al., 2017).

É importante considerar que o estudo foi realizado durante um cenário pandêmico, encontrando resultados que refletem a realidade deste contexto. Nesse sentido, fazem-se necessários novos estudos que monitorem a qualidade dos serviços da APS e seus respectivos atributos em períodos pós-controle da pandemia.

\section{CONCLUSÃO}

Através da utilização do instrumento PCATool-Brasil 2020 avaliou-se a qualidade da assistência à saúde de um município de Goiás como pouco orientada à APS e às necessidades de sua população. O estudo identificou falhas e acertos no cuidado fornecido aos pacientes, com o potencial de proporcionar o planejamento e a adoção de condutas preventivas e assistenciais mais eficazes. Além disso, buscou-se incentivar outras contribuições científicas nessa temática, pois é perceptível que a avaliação do desempenho na área da saúde mantém-se como parâmetro importante para que gestores possam conduzir suas ações, aprimorando o acesso e a qualidade de serviços de saúde à população.

\section{REFERÊNCIAS}

1. ANDERSON MIP, et al. Cobertura universal en salud, atención primaria y medicina familiar. Rev. Bras. Med. Fam. Comunidade, 2016; 12(1): 4-30.

2. DONABEDIAN A. Aspects of medical care administrations: specifying requirements for health care. Harvard University Press, 1973; 419-473.

3. FAUSTO MCR, MATTA GC. Atenção Primária à Saúde: histórico e perspectivas. In: MOROSINI MVGC, CORBO ADA (Org.). Modelos de atenção e a saúde da família. Fiocruz, 2007; 43-68.

4. HARZHEIM E, et al. Quality and effectiveness of different approaches to primary care delivery in Brazil. BMC Health Services Research, 2006; 6(1): 1-7. 
5. HARZHEIM E, et al. Validação do instrumento de avaliação da atenção primária à saúde: PCATool-Brasil adultos. Rev. Bras. Med. Fam. Comunidade, 2013; 8(29): 274-284.

6. HAUSER L, et al. Tradução, adaptação, validade e medidas de fidedignidade do instrumento de avaliação da atenção primária à saúde (PCATool) no Brasil: versão profissionais de saúde. Rev. Bras. Med. Fam. Comunidade, 2013; 8(29): 244-255.

7. MATTOS RA. Integralidade e a formulação de políticas específicas de saúde. In: PINHEIRO R, MATTOS RA de (Org). Construção da integralidade: cotidiano, saberes e práticas em saúde. Abrasco, 2007.

8. MENDES EV. As redes de atenção à saúde. Organização Pan-Americana de Saúde, 2011.

9. MINISTÉRIO DA SAÚDE. Secretaria de Atenção à Saúde. Departamento de Atenção Básica. Avaliação na Atenção Básica em Saúde: Caminhos da Institucionalização. Coordenação de Acompanhamento e Avaliação. 2005. Disponível em: https://www.nescon.medicina.ufmg.br/biblioteca/imagem/1521.pdf. Acessado em: 5 de março de 2021.

10. MINISTÉRIO DA SAÚDE. Secretaria Executiva. Departamento de Apoio à Descentralização. O SUS no seu município: garantindo saúde para todos. 2009.2 Disponível em: https://bvsms.saude.gov.br/bvs/publicacoes/sus_municipio_garantindo_saude.pdf. Acessado em: 5 de março de 2021.

11. MINISTÉRIO DA SAÚDE. Secretaria de Atenção à Saúde. Departamento de Atenção Básica. Política Nacional de

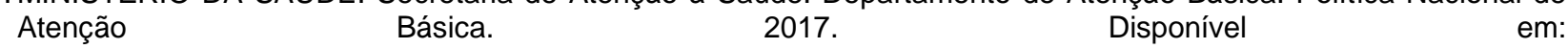
https://bvsms.saude.gov.br/bvs/saudelegis/gm/2017/prt2436_22_09_2017.html. Acessado em: 5 de março de 2021.

12. MINISTÉRIO DA SAÚDE. Secretaria de Atenção Primária à Saúde. Departamento de Saúde da Família. Manual do Instrumento de Avaliação da Atenção Primária à saúde: PCATool-Brasil-2020. 2020. Disponível em: https://www.conasems.org.br/wp-content/uploads/2020/05/Pcatool_2020.pdf. Acessado em: 5 de março de 2021.

13. NIED MM, et al. Elementos da atenção primária para compreender o acesso aos serviços do SUS diante do autorrelato do usuário. Cadernos Saúde Coletiva, 2020; 28: 362-372.

14. OLIVEIRA MAC, PEREIRA IC. Atributos essenciais da atenção primária e a estratégia saúde da família. Revista Brasileira de Enfermagem, 2013.

15. PENSO JM, et al. Avaliação da atenção primária à saúde utilizando o instrumento PCATool-Brasil. Rev. Bras. Med. Fam. Comunidade, 2017; 12(29): 1-9.

16. PIRES DA. Avaliação da gestão municipal da saúde bucal na atenção básica de municípios da região carbonífera de Santa Catarina: precisão do instrumento de pesquisa. Dissertação (Mestrado em Saúde Coletiva). Universidade Federal Santa Catarina, Florianópolis, 2012.

17. PUCCI, V.R. Avaliação do atributo da integralidade na atenção primária à saúde em municípios da região centrooeste no Rio Grande do Sul: pcatool-brasil versão profissionais. Dissertação (Mestrado, Pós-graduação em Enfermagem). Universidade Federal de Santa Maria, Santa Maria, 2017.

18. REIS RS, et al. Acesso e utilização dos serviços na estratégia saúde da família na perspectiva dos gestores, profissionais e usuários. Ciência \& Saúde Coletiva, 2013; 18(11): 3321-3331.

19. SCHMIDT MI, et al. Chronic non-communicable diseases in Brazil: burden and current challenges. The Lancet, 2011; 377(9781): 1949-1961.

20. SILVA KF. PCATool-Brasil versão profissionais: avaliação do atributo acesso do primeiro contato na atenção primária à saúde em município do interior do Rio Grande do Sul. Dissertação (Mestrado), Universidade Federal de Santa Maria, Santa Maria, 2017.

21. STARFIELD B. Atenção primária: equilíbrio entre necessidades de saúde, serviços e tecnologia. Brasília: Organização das Nações Unidas para a Educação, a Ciência e a Cultura. Ministério da Saúde, 2002.

22. STEIN AT. A avaliação dos serviços de saúde deve ser realizada com instrumentos validados. Epidemiol. Serv. Saúde, 2013; 22(1): 179-181.

23. TANAKA OU, TAMAKI EM. O papel da avaliação para a tomada de decisão na gestão de serviços de saúde. Ciênc. Saúde Coletiva, 2012; 17(4): 821-828. 